\title{
Recurrence of Susac Syndrome following 23 Years of Remission
}

\author{
Amalia Feresiadou $^{\mathrm{a}}$ Urban Eriksson $^{\mathrm{b}}$ Hans-Christian Larsen ${ }^{\mathrm{c}}$ \\ Raili Raininko $^{d}$ Ingela Nygren $^{a}$ Atle Melberg $^{a}$ \\ ${ }^{a}$ Neurology and ${ }^{b}$ Ophthalmology Units, Department of Neuroscience, 'Otolaryngology \\ Unit, Department of Surgical Sciences and ${ }^{\mathrm{d}}$ Radiology Unit, Department of Radiology, \\ Oncology and Radiation Science, Uppsala University, Uppsala, Sweden
}

\section{Key Words}

Branched retinal artery occlusion · Corpus callosum · Encephalopathy · Hearing loss . Recurrence $\cdot$ Retinal vasculopathy $\cdot$ Susac syndrome $\cdot$ Visual loss

\begin{abstract}
Susac syndrome is an autoimmune microangiopathy affecting the brain, retina and inner ear (cochlea and semicircular canals), leading to encephalopathy, branch retinal artery occlusions (BRAOs) and asymmetric neurosensory hearing loss, respectively. The natural history and long-term prognosis are variable as the disease has been shown to be monophasic and selflimiting, polycyclic or chronic continuous. We describe a 35-year-old woman who presented with a sudden hearing loss in the left ear in the 37th week of her second pregnancy. She subsequently developed BRAO in the right eye 2.5 months after having given birth. MRI findings included round lesions in the corpus callosum which are pathognomonic for Susac syndrome. Previous patient records documented encephalopathy, sudden deafness of the right ear and visual field defects in the left eye at the age of 12 , followed by permanent hearing and visual defects. We expand on the variability in the course of Susac syndrome as recurrence may occur after as long as 23 years. Cases of monophasic self-limiting Susac syndrome may in fact turn polycyclic with an interval of more than 2 decades between the bouts of the disease. In these cases, suspecting the development of exacerbation early is important in order to start the treatment promptly.

(c) 2014 S. Karger AG, Basel
\end{abstract}

\section{Introduction}

Susac syndrome was first described in 1979 [1] and it is a vasculopathy affecting the retina, inner ear and the brain [2]. Up to now, more than 300 cases have been reported [3].

Amalia Feresiadou, MD

Department of Neurology

Uppsala University Hospital

SE-75185 Uppsala (Sweden)

E-Mail feresiadouamalia@gmail.com 
Feresiadou et al.: Recurrence of Susac Syndrome following 23 Years of Remission

The disease is considered an autoimmune endotheliopathy [4,5]. Anti-endothelial cell antibodies have been demonstrated in patients with Susac syndrome $[5,6]$, but larger studies need to be done to confirm their specificity and thus their value as a diagnostic tool [3].

The clinical triad of encephalopathy, branch retinal artery occlusion (BRAO) and hearing loss is typical for the disease. All 3 features may not be present at the onset, which complicates the diagnosis of Susac syndrome. Hearing loss and visual loss are asymmetrical. MRI show a distinctive pattern of supratentorial white matter lesions that always involve the central corpus callosum [7]. Our understanding of the natural history of Susac syndrome is incomplete [8]. Immunosuppressive treatment is indicated during active stages of the disease. We report a case of Susac syndrome with recurrence of encephalopathy, hearing loss and BRAO 23 years after the first occurrence.

\section{Case Presentation}

A 35-year-old-woman presented in the 37th week of her second pregnancy with a relatively sudden hearing loss and tinnitus in the left ear. Initially, the symptoms were fluctuating. She suffered from attacks of vertigo and slight difficulty in finding words. Approximately 2.5 months after having given birth, she developed painless visual acuity impairment and noticed a dark shadow in the inferior visual field of the right eye. Clinical fundoscopy examination revealed an occlusion of the superior retinal arteries and a pale ischemic edema of the retina in the upper quadrants (fig. 1a). In the left eye, a pale optic nervehead, thin arterial white 'ghost vessels' and an atrophic retina were observed as is usually seen after old ischemic catastrophes (fig. 1b). Approximately 2 weeks later, visual field testing showed a total-field defect in the right eye inferiorly and a small-field remnant in the upper temporal quadrant of the left eye. The visual field did not improve but the visual acuity did to some degree. Clinical examination was normal apart from hearing loss and visual defects.

A psychometric test, routine blood tests, erythrocyte sedimentation rate, serum lipids, blood glucose, creatinine, complement and coagulation screening, anti-neutrophil antibodies, anti-nuclear cytoplasmic antibodies, cardiolipin antibodies, electrocardiogram, echocardiography and Doppler ultrasound examination of the precerebral arteries were all normal. Cerebrospinal fluid was normal regarding cells, albumin and IgG index, and there were no oligoclonal bands. Pure tone audiometry (PTA) revealed a mild hearing loss in the left ear at $26.2 \mathrm{~dB}$. The speech discrimination rates were $28 \%$ in the right ear and $78 \%$ in the left ear. Acoustic brainstem responses could be measured in both ears at 70 and $90 \mathrm{~dB}$ (click stimulation, Ergometric). Wave I was absent in both ears, wave III could be identified in the left ear and wave $\mathrm{V}$ could be reproduced bilaterally with both normal configuration and symmetrical normal latency. Stapedial reflexes were registered at stimulation with $90 \mathrm{~dB}$ in the left ear at 500-1,000-2,000 $\mathrm{Hz}$ and recorded in the right ear with contralateral stimulation of the left ear. MRI revealed supratentorial white matter lesions including round lesions in the corpus callosum (fig. 2a-d). EEG showed intermittent delta activity in the frontotemporal region bilaterally.

The diagnosis of Susac syndrome was made in the period after the patient had given birth, when she developed BRAO in addition to the hearing loss. MRI confirmed the diagnosis. The patient commenced treatment with acetylsalicylic acid $75 \mathrm{mg} /$ day and received methylprednisolone $1,000 \mathrm{mg}$ intravenously daily for 5 days and thereafter prednisolone $80 \mathrm{mg} /$ day. Prednisolone was tapered subsequently as she received cyclophosphamide $100 \mathrm{mg} /$ day, which was discontinued after 5 months. She continued treatment 
with prednisolone $5 \mathrm{mg}$ /day and acetylsalicylic acid, and prednisolone was further decreased to $5 \mathrm{mg}$ every other day. On this regimen, the condition has been stable regarding hearing and vision for the past 3 years. She has not suffered from migraine attacks since commencing immunosuppressive treatment.

We reviewed the patient's medical charts. Since the age of 11 , she had suffered from migraine headaches with visual aura occurring about once a week. At the age of 12 , she experienced an abrupt hearing loss in the right ear associated with tiredness, nausea and vomiting. According to relatives, her short-term memory had allegedly also been affected. Several PTA measurements showed a sensorineural hearing loss for low to middle frequencies in the right ear resulting in a profound hearing loss with a PTA of $73 \mathrm{~dB}$. EEG showed slow background theta activity and intermittent rhythmic delta activity in the precentral areas. Later that same year, she noted partial visual loss in the left eye. CT of the brain, performed twice, was normal. Apart from a hearing loss and visual defects, her clinical examination was normal. The hearing loss was permanent bilaterally, mostly in the right ear in which she wore a hearing aid. Perimetry revealed a visual field defect inferiorly in the left eye, which had progressed during the first 4 years of follow-up but has remained unchanged thereafter. The patient did not receive immunosuppressive treatment. She developed a pale atrophic optic nerve. She also had a long history of headaches judged as tension-type headache and migraine with visual aura but reported no subjective episodes of deterioration of hearing or vision until the age of 35 .

\section{Discussion}

Susac syndrome has been reported to be monophasic and self-limiting, without recurrence after remission [2, 9], polycyclic or rarely chronic continuous [8]. Disease activity may occur over several years with fluctuating symptoms, and relapses of isolated BRAOs may occur after several years $[4,10]$. The disease may be disabling, protracted and severe in some cases [11] and rarely leading to death [12]. Petty et al. [13] reported a 31-year-old pregnant woman who developed encephalopathy, a hearing loss and segmental visual loss. EEG documented delta activity bilaterally. The patient then developed memory loss as well as hearing and visual losses. Eighteen years later, after having commenced estrogen replacement therapy, she deteriorated with encephalopathy, hearing loss and BRAO.

We regard our case and the one reported by Petty et al. [13] as examples of the polycyclic nature of Susac syndrome. In our patient, immunosuppressive treatment was not administered at the age of 12 , and the disease was self-limiting. Treatment was started at the age of 35 in the period after she had given birth. Headache, often of migraine character, is a common feature in Susac syndrome [3]. After starting immunosuppressive treatment, our patient's migraine has disappeared altogether. Overall, she has been stable for the past 3 years regarding neurological symptoms, vision and hearing.

It is unknown what triggers episodes of Susac syndrome. The syndrome has a strong female preponderance with a 3.5:1 female/male ratio [3]. It has been reported that $5 \%$ of female patients develop symptoms during pregnancy or the period after giving birth [3], as in the recurrence of Susac syndrome in our patient.

There are no controlled studies on the treatment of Susac syndrome. Early treatment with immunosuppressive agents and possibly antiplatelet drugs is recommended during active stages of the disease with the goal to minimize permanent visual, hearing and brain impairment [3]. Several treatment regimens have been suggested $[4,11,14]$. 
We report that Susac syndrome may recur after as many as 23 years since remission, thus expanding the knowledge on the natural course of the illness. The significance of this observation is that apparently monophasic self-limiting Susac syndrome may in fact recur after an interval longer than 2 decades, thus being polycyclic. It is therefore important to consider a new bout of the disease and treatment early after recurrence, even before the complete triad of hearing loss, BRAO and encephalopathy has developed.

\section{Acknowledgement}

This study was financially supported by the Selander foundation and Uppsala University.

\section{Disclosure Statement}

The authors declare no conflicts of interest.

\section{References}

1 Susac JO, Hardman JM, Selhorst JB: Microangiopathy of the brain and retina. Neurology 1979;29:313-316.

12 Susac JO: Susac's syndrome: the triad of microangiopathy of the brain and retina with hearing loss in young women. Neurology 1994;44:591-593.

-3 Dörr J, Krautwald S, Wildemann B, Jarius S, Ringelstein M, Duning T, Aktas O, Ringelstein EB, Paul F, Kleffner I: Characteristics of Susac syndrome: a review of all reported cases. Nat Rev Neurol 2013; 9:307-316.

4 Rennebohm RM, Egan RA, Susac JO: Treatment of Susac's syndrome. Curr Treat Options Neurol 2008;10:6774.

$>5$ Jarius S, Neumayer B, Wandinger KP, Hartmann M, Wildemann B: Anti-endothelial serum antibodies in a patient with Susac's syndrome. J Neurol Sci 2009;285:259-261.

-6 Magro CM, Poe JC, Lubow M, Susac JO: Susac syndrome: an organ-specific autoimmune endotheliopathy syndrome associated with anti-endothelial cell antibodies. Am J Clin Pathol 2011;136:903-912.

7 Susac JO, Murtagh FR, Egan RA, Berger JR, Bakshi R, Lincoff N, Gean AD, Galetta SL, Fox RJ, Costello FE, Lee AG, Clark J, Layzer RB, Daroff RB: MRI findings in Susac's syndrome. Neurology 2003;61:1783-1787.

8 Susac JO, Egan RA, Rennebohm RM, Lubow M: Susac's syndrome: 1975-2005 microangiopathy/autoimmune endotheliopathy. J Neurol Sci 2007;257:270-272.

-9 Petty GW, Engel AG, Younge BR, Duffy J, Yanagihara T, Lucchinetti CF, Bartleson JD, Parisi JE, Kasperbauer JL, Rodriguez M: Retinocochleocerebral vasculopathy. Medicine (Baltimore) 1998;77:12-40.

-10 Aubart-Cohen F, Klein I, Alexandra JF, Bodaghi B, Doan S, Fardeau C, Lavallée P, Piette JC, Hoang PL, Papo T: Long-term outcome in Susac syndrome. Medicine (Baltimore) 2007;86:93-102.

11 Mateen FJ, Zubkov AY, Muralidharan R, Fugate JE, Rodriguez FJ, Winters JL, Petty GW: Susac syndrome: clinical characteristics and treatment in 29 new cases. Eur J Neurol 2012;19:800-811.

-12 Saux A, Niango G, Charif M, Morales R, Mura F, Bonafe A, Mourand I: Susac's syndrome, a rare, potentially severe or lethal neurological disease. J Neurol Sci 2010;297:71-73.

13 Petty GW, Matteson EL, Younge BR, McDonald TJ, Wood CP: Recurrence of Susac syndrome (retinocochleocerebral vasculopathy) after remission of 18 years. Mayo Clin Proc 2001;76:958-960.

14 Rennebohm R, Susac JO, Egan RA, Daroff RB: Susac's Syndrome - update. J Neurol Sci 2010;299:86-91. 


\section{Case Reports in Neurology}

\begin{tabular}{l|l}
\hline \multicolumn{2}{l}{ Case Rep Neurol 2014;6:171-175 } \\
\hline DOI: 10.1159/000362868 & $\begin{array}{l}\text { (C) 2014 S. Karger AG, Basel } \\
\text { www.karger.com/crn }\end{array}$ \\
\hline
\end{tabular}

Feresiadou et al.: Recurrence of Susac Syndrome following 23 Years of Remission

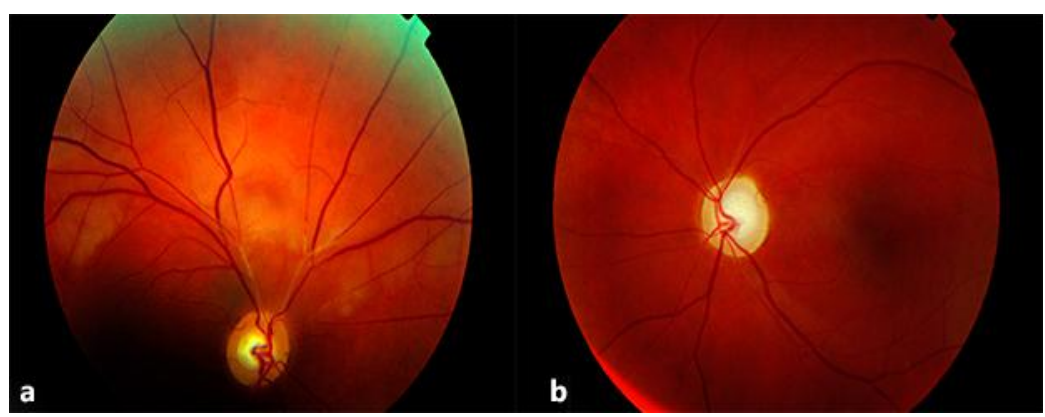

Fig. 1. a Right fundus. A normal optic nerve with segmental obstructions of the arteries in the temporal and nasal superior branches. A pale ischemic edema is present in the retina superior to the optic nerve. $\mathbf{b}$ Left fundus. A pale atrophic optic nervehead and thin white 'ghost vessels' were observed in the superior temporal arcade.
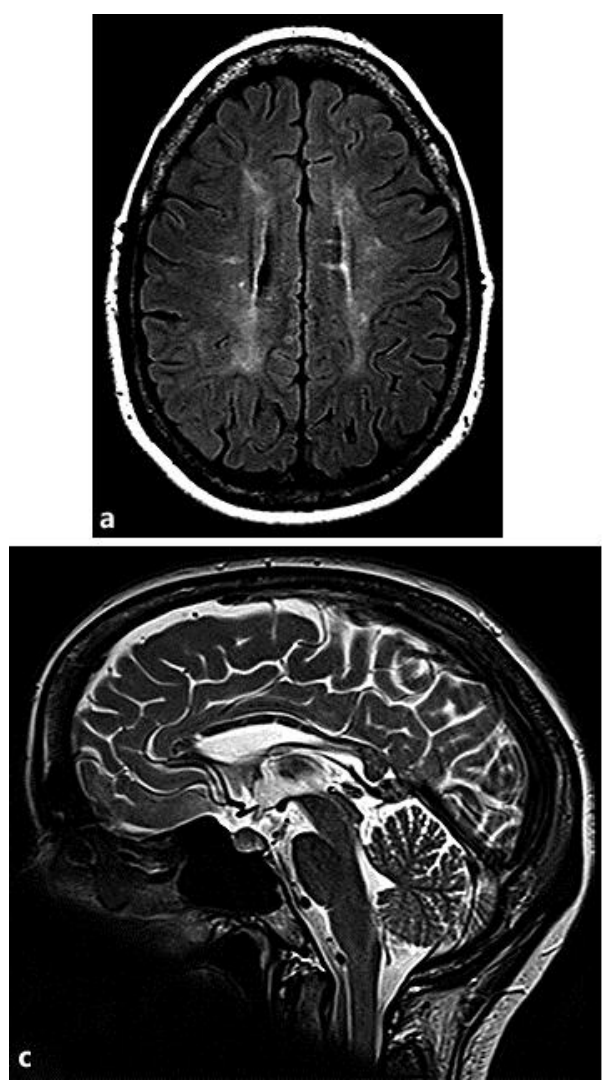
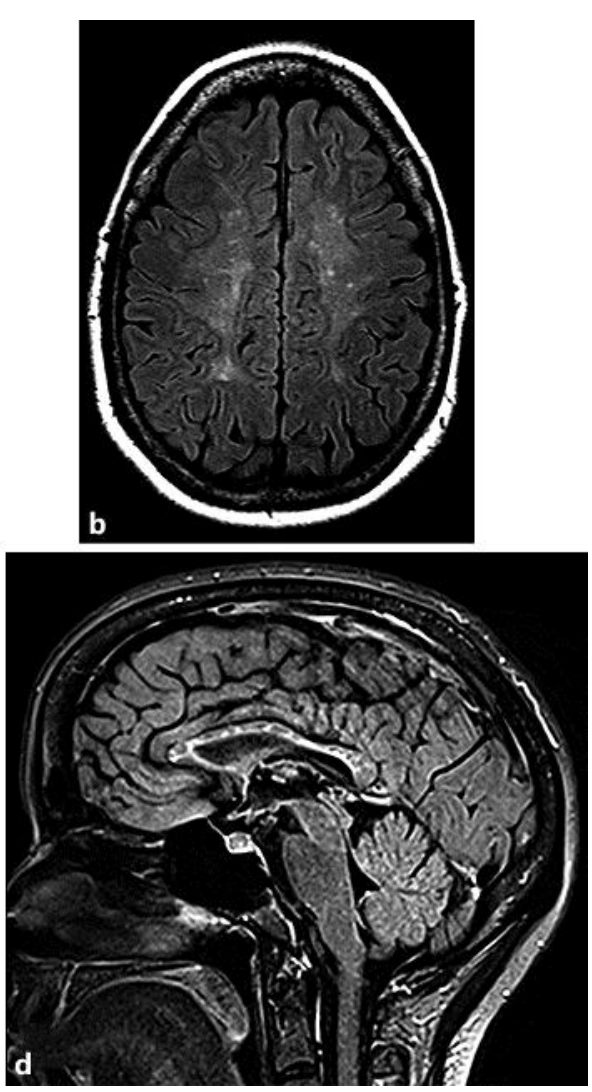

Fig. 2. T2-weighted MR images of the brain. a, b Transverse images with a FLAIR sequence show high signal intensity changes in white matter. c A sagittal image with a spin echo sequence demonstrates multiple round lesions in the corpus callosum. $\mathbf{d}$ The same slice with a FLAIR sequence reveals that not only the nodules seen in $\mathbf{c}$ but also large areas in the corpus callosum emit a pathological high signal. 